\title{
Infinite Hilbert 2-class field tower of quadratic number fields
}

by

\section{A. Mounib (Taza)}

1. Introduction. Let $k$ be a number field. We will denote the ideal class group of $k$ in the wide sense by $C_{k}$. Let $k^{1}$ be the Hilbert 2-class field of $k$ (i.e., the maximal abelian unramified 2-extension of $k$ ), and for $n \geq 2$, let $k^{n}$ be the Hilbert 2-class field of $k^{n-1}$. Then

$$
k \subset k^{1} \subset k^{2} \subset \cdots \subset k^{n} \subset \cdots
$$

is the Hilbert 2-class field tower of $k$. We say that the tower is finite if $k^{n}=k^{n+1}$ for some $n$, and infinite otherwise.

We define the 2-rank of $C_{k}$ as the dimension of the elementary abelian 2-group $C_{k} / C_{k}^{2}$ viewed as a vector space over $\mathbb{F}_{2}$ :

$$
\operatorname{rank}_{2}\left(C_{k}\right)=\operatorname{dim}_{\mathbb{F}_{2}}\left(C_{k} / C_{k}^{2}\right),
$$

where $\mathbb{F}_{2}$ is the finite field with two elements. We define the 4 -rank of $C_{k}$ by

$$
\operatorname{rank}_{4}\left(C_{k}\right)=\operatorname{rank}_{2}\left(C_{k}^{2}\right)=\operatorname{dim}_{\mathbb{F}_{2}}\left(C_{k}^{2} / C_{k}^{4}\right) .
$$

Assume $k$ is an imaginary quadratic number field. It is well known that if $\operatorname{rank}_{2}\left(C_{k}\right) \geq 5$, then the Hilbert 2-class field tower of $k$ is infinite [5]. In the case where $\operatorname{rank}_{2}\left(C_{k}\right)=2$ or 3 , the Hilbert 2-class field tower of $k$ may be finite $([9],[10])$, and if $\operatorname{rank}_{2}\left(C_{k}\right)=1$ then the Hilbert 2-class field tower of $k$ is finite of length 1 . It has been conjectured that if $\operatorname{rank}_{2}\left(C_{k}\right)=4$, then $k$ has infinite Hilbert 2-class field tower [10]. We mention that Hajir proved that if $C_{k}$ contains a subgroup isomorphic to $\mathbb{Z} / 4 \mathbb{Z} \times \mathbb{Z} / 4 \mathbb{Z} \times \mathbb{Z} / 4 \mathbb{Z}$, then $k$ has infinite Hilbert 2-class field tower ([6], [7]).

Now suppose that $\operatorname{rank}_{2}\left(C_{k}\right)=4$ and the discriminant of $k$ is divisible by exactly one negative prime discriminant. In [2], under some conditions on the 4-rank of $C_{k}$ and the Kronecker symbols of the primes dividing the discriminant of $k$, the author proves that $k$ has infinite Hilbert 2-class field tower. Y. Sueyoshi proves the same result under some conditions on the Rédei matrix [14]. 
In Section 3 of this article, we investigate Martinet's question and the above conjecture by generalizing the preceding results. We prove the following theorem:

TheOREM. Let $k$ be an imaginary quadratic number field whose discriminant is divisible by at most one negative prime discriminant and $\operatorname{rank}_{2}\left(C_{k}\right)$ $=4$. Then the Hilbert 2-class field tower of $k$ is infinite.

Also, in Section 3, we show that a positive proportion of imaginary quadratic number fields with the class group of 2-rank equal to 2 and 4-rank equal to 1 have infinite Hilbert 2-class field towers.

\section{Known results}

2.1. Golod and Shafarevich inequality. Let $k$ be a number field, $C_{k}$ be the class group of $k$ and $E_{k}$ be the group of units of $k$. Then, from [3, p. 233], we know that the Hilbert 2-class field tower of $k$ is infinite if

$$
\operatorname{rank}_{2}\left(C_{k}\right) \geq 2+2 \sqrt{\operatorname{rank}_{2}\left(E_{k}\right)+1}
$$

where $\operatorname{rank}_{2}\left(E_{k}\right)$ is exactly the number of infinite primes of $k$.

REMARKS. If $k$ is an imaginary quadratic number field, then $\operatorname{rank}_{2}\left(E_{k}\right)$ $=1$. Suppose $\operatorname{rank}_{2}\left(C_{k}\right) \geq 5$. Then the inequality $(*)$ is satisfied and $k$ has infinite Hilbert 2-class field tower.

If $k$ is an imaginary biquadratic number field, then $\operatorname{rank}_{2}\left(E_{k}\right)=2$. Suppose $\operatorname{rank}_{2}\left(C_{k}\right) \geq 6$. Then the inequality $(*)$ is satisfied and $k$ has infinite Hilbert 2-class field tower.

If $k$ is an imaginary triquadratic number field, then $\operatorname{rank}_{2}\left(E_{k}\right)=4$, and the inequality $(*)$ is satisfied whenever $\operatorname{rank}_{2}\left(C_{k}\right) \geq 7$.

2.2. Genus theory. Let $K$ be a quadratic extension of a number field $k$. By classical results of genus theory [8], we have

$$
\operatorname{rank}_{2}\left(C_{K}\right) \geq \operatorname{ram}(K / k)-\operatorname{dim}_{\mathbb{F}_{2}}\left(E_{k} / E_{k} \cap N_{K / k}\left(K^{*}\right)\right)-1,
$$

where $\operatorname{ram}(K / k)$ is the number of primes that ramify in the extension $K / k$, and $N_{K / k}$ is the norm map in the extension $K / k$. In the case where the class number of $k$ is odd, the preceding inequality becomes an equality (see for instance [1]).

We note that

$$
\operatorname{dim}_{\mathbb{F}_{2}}\left(E_{k} / E_{k} \cap N_{K / k}\left(K^{*}\right)\right) \leq \begin{cases}{[k: \mathbb{Q}]} & \text { if } k \text { totally real, } \\ \frac{1}{2}[k: \mathbb{Q}] & \text { if not. }\end{cases}
$$

Now let $k$ be a quadratic number field of discriminant $d$, and $t$ be the number of primes that ramify in $k$. By genus theory, we have

$$
\operatorname{rank}_{2}\left(C_{k}\right)= \begin{cases}t-2 & \text { if } d \text { is positive and not a sum of two squares, } \\ t-1 & \text { otherwise. }\end{cases}
$$




\section{Main results}

3.1. Proof of the Theorem. We let the notations be as in Section 2. In this section we investigate Martinet's conjecture, we give a proof of the Theorem and we show that a positive proportion of some imaginary quadratic number fields have infinite Hilbert 2-class field tower. We begin with the following two lemmas.

Lemma 3.1. Let $p_{1}, p_{2}, p_{3}$ and $p_{4}$ be distinct prime numbers $\not \equiv-1$ $(\bmod 4)$ and $K=\mathbb{Q}\left(\sqrt{p_{1}}, \sqrt{p_{2}}, \sqrt{p_{3}}, \sqrt{p_{4}}\right)$. Then $\operatorname{rank}_{2}\left(C_{K}\right) \geq 2$.

Proof. See [11, Theorem 5.3].

Lemma 3.2. Let $p$ be a prime number and $L / K$ be a Galois extension of algebraic number fields whose Galois group $G$ is an elementary p-group. Then for each place $\mathcal{P}$ of $K$ unramified in $L$, the number of $\mathcal{P}$-places of $L$ is equal to $[L: K]$ or $(1 / p)[L: K]$.

Proof. We know that if $\mathcal{P}$ is unramified in the extension $L / K$, then the decomposition group of $\mathcal{P}$ is a cyclic subgroup of $G$. Since $G$ is an elementary $p$-group, the decomposition group of $\mathcal{P}$ is of order 1 or $p$, proving the lemma.

Proof of the Theorem. By hypotheses, we have $\operatorname{rank}_{2}\left(C_{k}\right)=4$ and the discriminant $d$ of $k$ is divisible by at most one prime $\equiv-1(\bmod 4)$. So, denote by $p_{1}, p_{2}, p_{3}, p_{4}$ and $p$ distinct prime numbers dividing $d$ such that $p_{i} \not \equiv-1(\bmod 4), 1 \leq i \leq 4$ and $p=2$ or $p \equiv-1(\bmod 4)$. We put $K=\mathbb{Q}\left(\sqrt{p_{1}}, \sqrt{p_{2}}, \sqrt{p_{3}}, \sqrt{p_{4}}\right)$ and let $M$ be the decomposition field of $p$ in $K$. From Lemma $3.2, M=K$ or $K / M$ is a quadratic extension. Let $F$ be the composite field of $M$ and $k$ which is a totally complex quadratic extension of the totally real field $M$.

Suppose that $M=K$. Then the extension $F / M$ is ramified at all archimedian places and $p$-adic places of $M$, so $\operatorname{ram}(F / M)=2[M: \mathbb{Q}]=32$. We have $\operatorname{dim}_{\mathbb{F}_{2}}\left(E_{M} / E_{M} \cap N_{F / M}\left(F^{*}\right)\right) \leq[M: \mathbb{Q}]$ and $\operatorname{rank}_{2}\left(E_{F}\right)=[M: \mathbb{Q}]$ $=16$. Hence one can readily verify that

$$
\operatorname{ram}(F / M)-\operatorname{dim}_{\mathbb{F}_{2}}\left(E_{M} / E_{M} \cap N_{F / M}\left(F^{*}\right)\right)-1 \geq 2+2 \sqrt{\operatorname{rank}_{2}\left(E_{F}\right)+1} .
$$

By Section 2.2, we have

$$
\operatorname{rank}_{2}\left(C_{F}\right) \geq \operatorname{ram}(F / M)-\operatorname{dim}_{\mathbb{F}_{2}}\left(E_{M} / E_{M} \cap N_{F / M}\left(F^{*}\right)\right)-1,
$$

so the extension $F / M$ satisfies the inequality $(*)$ of Section 2.1 , and consequently $F$ has infinite Hilbert 2 -class field tower. Therefore, since $F / k$ is unramified, $k$ has infinite Hilbert 2-class field tower.

Suppose that $K / M$ is a quadratic extension. In the case where $K / M$ is ramified, there exists a unique $i \in\{1,2,3,4\}$ such that the $p_{i}$-adic places 
of $M$ are ramified in $K$. So the extension $F / M$ is ramified at all archimedian places, $p$-adic places and $p_{i}$-adic places of $M$. Moreover, we have $\operatorname{ram}(F / M)=3[M: \mathbb{Q}]=24$ or $\operatorname{ram}(F / M)=2[M: \mathbb{Q}]+\frac{1}{2}[M: \mathbb{Q}]=20$ respectively if $p_{i}$ is totally decomposed in $M$ or not. Therefore, as in the preceding case, we show that the Hilbert 2-class tower of $k$ is infinite. It remains to study the case where $K / M$ is an unramified quadratic extension.

Suppose that $K / M$ is unramified. By Lemma 3.1 we have $\operatorname{rank}_{2}\left(C_{K}\right) \geq 2$, so the 2-part of the class group of $M$ can never be trivial or cyclic. This implies that $\operatorname{rank}_{2}\left(C_{M}\right) \geq 2$. Let $\tilde{M}$ be the maximal elementary unramified extension of $M$. One can verify that $\tilde{M}$ is normal over $\mathbb{Q}$. Denote by $F$ the composite field of $\tilde{M}$ and $k$ which is a totally complex quadratic extension of the totally real field $\tilde{M}$. The extension $F / \tilde{M}$ is ramified at all archimedian places and $p$-adic places of $\tilde{M}$. By Lemma 3.2, each $p$-adic place of $M$ is totally decomposed or decomposed into $\frac{1}{2}[\tilde{M}: M]$ places in $\tilde{M}$, so $\operatorname{ram}(F / \tilde{M}) \geq[\tilde{M}: \mathbb{Q}]+\frac{1}{2}[\tilde{M}: \mathbb{Q}]$. On the other hand since $\operatorname{rank}_{2}\left(E_{F}\right)=[\tilde{M}: \mathbb{Q}]$ and $[\tilde{M}: \mathbb{Q}] \geq 32$, one can obtain

$$
\operatorname{ram}(F / \tilde{M})-\operatorname{dim}_{\mathbb{F}_{2}}\left(E_{\tilde{M}} / E_{\tilde{M}} \cap N_{F / \tilde{M}}\left(F^{*}\right)\right)-1 \geq 2+2 \sqrt{\operatorname{rank}_{2}\left(E_{F}\right)+1},
$$

hence the extension $F$ satisfies the inequality $(*)$, and consequently $F$ has infinite Hilbert 2-class field tower. The fact that $F / k$ is unramified implies that $k$ has infinite Hilbert 2-class field tower.

3.2. The positive proportion of imaginary quadratic number fields $k$ with 2-rank of $C_{k}$ equal to 2. It is well known that every number field whose 2 -part of its class group is isomorphic to $\mathbb{Z} / 2 \mathbb{Z} \times \mathbb{Z} / 2 \mathbb{Z}$ has finite Hilbert 2-class field tower that terminates in at most two steps [9].

In [10], J. Martinet asked the following question: is there any imaginary quadratic number field with 2-class group of rank 2 and infinite Hilbert 2-class field tower?

Schmithals showed that the quadratic number field $k=\mathbb{Q}(\sqrt{-25355})$ with $\operatorname{rank}_{2}\left(C_{k}\right)=2$ has infinite Hilbert 2-class field tower [13].

In the following proposition we show that a positive proportion of imaginary quadratic number fields with 2-rank of its class group equal to 2 and its 4-rank equal to 1 have infinite Hilbert 2-class field towers.

Proposition 3.3. Let $p_{1}$ and $p_{2}$ be distinct prime numbers such that the class number of $\mathbb{Q}\left(\sqrt{p_{1} p_{2}}\right)$ is divisible by 16 . Then for each prime number $p \equiv-1(\bmod 4)$ such that $\left(\frac{p_{1} p_{2}}{p}\right)=-1$, the Hilbert 2 -class field tower of $\mathbb{Q}\left(\sqrt{-p_{1} p_{2} p}\right)$ is infinite.

Proof. From genus theory, the 2-class group of $k=\mathbb{Q}\left(\sqrt{p_{1} p_{2}}\right)$ is cyclique. Since by hypotheses, the class number of $k$ is divisible by 4 , we have $\left(\frac{p_{1}}{p_{2}}\right)=1$ [12]. Moreover, $\left(\frac{p_{1} p_{2}}{p}\right)=-1$ and thus the Rédei matrix of 
$\mathbb{Q}\left(\sqrt{-p_{1} p_{2} p}\right)$ has rank 1 , which implies that the 4-rank of the class group of $\mathbb{Q}\left(\sqrt{-p_{1} p_{2} p}\right)$ is equal to 1 [4]. Now let $k^{1}$ be the Hilbert 2-class field of $k$ and $F$ be the composite field of $k^{1}$ and $\mathbb{Q}(\sqrt{-p})$ which is a totally complex quadratic extension of the totally real field $k^{1}$. It is clear that $F / \mathbb{Q}\left(\sqrt{-p_{1} p_{2} p}\right)$ is unramified. Then proving the theorem is reduced to proving that $F$ has infinite Hilbert 2-class field tower.

The prime number $p$ is inert in the extension $k / \mathbb{Q}$, since $\left(\frac{p_{1} p_{2}}{p}\right)=-1$. Thus the $p$-adic place of $k$ is principal. So by the reciprocity law applied in the extension $k^{1} / k$, the $p$-adic place of $k$ is totally decomposed in $k^{1}$. Note that the number of $p$-adic places that ramify in $F / k^{1}$ is equal to $\left[k^{1}: k\right]$. Thus $\operatorname{ram}\left(F / k^{1}\right)=3\left[k^{1}: k\right]$. From Section 2.2, we have

$$
\operatorname{rank}_{2}\left(C_{F}\right) \geq \operatorname{ram}\left(F / k^{1}\right)-\operatorname{dim}_{\mathbb{F}_{2}}\left(E_{k^{1}} / E_{k^{1}} \cap N_{F / k^{1}}\left(F^{*}\right)\right)-1
$$

and since $\operatorname{dim}_{\mathbb{F}_{2}}\left(E_{k^{1}} / E_{k^{1}} \cap N_{F / k^{1}}\left(F^{*}\right)\right) \leq 2\left[k^{1}: k\right]$, it follows that $\operatorname{rank}_{2}\left(C_{F}\right)$ $\geq\left[k^{1}: k\right]-1 \geq 15$. On the other hand, since $\operatorname{rank}_{2}\left(E_{F}\right)=2\left[k^{1}: k\right]$ and one can verify that $\left[k^{1}: k\right]-1 \geq 2+2 \sqrt{2\left[k^{1}: k\right]+1}$, by the inequality $(*)$ of Section 2.1 we deduce that the Hilbert 2-class field tower of $F$ is infinite. Hence $\mathbb{Q}\left(\sqrt{-p_{1} p_{2} p}\right)$ has infinite Hilbert 2-class field tower.

By the distribution of prime numbers in an arithmetic progression, there exist infinitely many primes $p$ satisfying the conditions of the preceding proposition. Thus the proposition shows that a positive proportion of the imaginary quadratic number fields with 2-rank of the class group equal to 2 and 4-rank equal to 1 have infinite Hilbert 2-class field towers.

From the following proposition we construct imaginary quadratic number fields $k$ such that $\operatorname{rank}_{2}\left(C_{k}\right)=\operatorname{rank}_{4}\left(C_{k}\right)=2$ and $k$ has infinite Hilbert 2-class field tower.

Proposition 3.4. Let $d$ be a positive integer such that $d \not \equiv 1(\bmod 4)$ and $k=\mathbb{Q}(\sqrt{d})$. Suppose that 8 divides the order of $C_{k}$. Then for every prime number $p \equiv-1(\bmod 4)$ such that the equation $x^{2}-d y^{2}=p$ has a solution in $\mathbb{Z} \times \mathbb{Z}$, the imaginary quadratic number field $\mathbb{Q}(\sqrt{-p d})$ has infinite Hilbert 2-class field tower.

Proof. The equation $x^{2}-d y^{2}=p$ having a solution in $\mathbb{Z} \times \mathbb{Z}$ implies that $p$ is decomposed into two distinct primes $\mathcal{P}_{1}$ and $\mathcal{P}_{2}$ in $k$. We have $p o_{k}=\mathcal{P}_{1} \mathcal{P}_{2}=(a-b \sqrt{d})(a+b \sqrt{d}) o_{k}$ where $a$ and $b$ are two positive integers and $o_{k}$ the ring of integers of $k$. Then the places $\mathcal{P}_{1}$ and $\mathcal{P}_{2}$ are principal. Therefore, $\mathcal{P}_{1}$ and $\mathcal{P}_{2}$ are totally decomposed in the Hilbert 2-class field $k^{1}$ of $k$, so $p$ is totally decomposed in $k^{1}$. The extension $k^{1}(\sqrt{-p}) / k^{1}$ is ramified at the archimedian and the $p$-adic places of $k^{1}$, hence it is easy to see that $k^{1}(\sqrt{-p})$ satisfies the equality $(*)$, so the Hilbert 2 -class field tower of $k^{1}(\sqrt{-p})$ is infinite. The fact that $k^{1}(\sqrt{-p}) / \mathbb{Q}(\sqrt{-p d})$ is unramified proves the example. 
Let $d=226$ and $p=367$. The class number of $k=\mathbb{Q}(\sqrt{d})$ is equal to 8 . Since $49^{2}-3^{2} d=p$, from the preceding proposition $\mathbb{Q}(\sqrt{-p d})$ has infinite Hilbert 2-class field tower.

Let $d=226$ and $p=503$. The class number of $k=\mathbb{Q}(\sqrt{d})$ is equal to 8 . Since $27^{2}-d=p$, from the preceding proposition $\mathbb{Q}(\sqrt{-p d})$ has infinite Hilbert 2-class field tower.

\section{References}

[1] A. Azizi et A. Mouhib, Sur le rang du 2-groupe de classes de $\mathbb{Q}(\sqrt{m}, \sqrt{d})$ où $m=2$ ou un premier $p \equiv 1(\bmod 4)$, Trans. Amer. Math. Soc. 353 (2001), 2741-2752.

[2] E. Benjamin, On imaginary quadratic number fields with 2-class group of rank 4 and infinite 2-class field tower, Pacific J. Math. 201 (2001), 257-266.

[3] J. W. S. Cassels and A. Fröhlich, Algebraic Number Theory, Academic Press, London, 1986.

[4] F. Gerth, The 4-class ranks of quadratic fields, Invent. Math. 77 (1984), 489-515.

[5] E. S. Golod and I. R. Shafarevich, On the class field tower, Izv. Akad. Nauk SSSR Ser. Mat. 28 (1964), 261-272 (in Russian); English transl.: Amer. Math. Soc. Transl. 48 (1965), 91-102.

[6] F. Hajir, On a theorem of Koch, Pacific J. Math. 176 (1996), 15-18.

[7] —, Correction to "On a theorem of Koch", ibid. 196 (2000), 507-508.

[8] W. Jehne, On knots in algebraic number theory, J. Reine Angew. Math. 311/312 (1979), 215-254.

[9] H. Kisilevsky, Number fields with class number congruent to 4 mod 8 and Hilbert's theorem 94, J. Number Theory 8 (1976), 271-279.

[10] J. Martinet, Tours de corps de classes et estimations de discriminants, Invent. Math. 44 (1978), 65-73.

[11] A. Mouhib et A. Movahhedi, Sur le 2-groupe de classes des corps multiquadratiques réels, J. Théor. Nombres Bordeaux 17 (2005), 619-641.

[12] L. Rédei und H. Reichardt, Die Anzahl der durch 4 teilbaren Invarianten der Klassengruppe eines beliebigen quadratischen Zahlkörpers, J. Reine Angew. Math. 170 (1933), 69-74.

[13] B. Schmithals, Konstruktion imaginärquadratischer Körper mit unendlichem Klassenkörperturm, Arch. Math. (Basel) 34 (1980), 307-312.

[14] Y. Sueyoshi, Infinite 2-class field towers of some imaginary quadratic number fields, Acta Arith. 113 (2004), 251-257.
A. Mouhib
Laboratoire Informatique, Mathématique, Automatique et Optoélectronique
Faculté polydisciplinaire
Université Mohamed Ben Abdellah
B/P 1223, Taza-Gare, Maroc
E-mail: mouhibali@yahoo.fr. 